\title{
ON THE STABILITY OF A GENERAL MAGNETIC FIELD TOPOLOGY IN STELLAR RADIATIVE ZONES
}

\author{
K. Augustson ${ }^{1}$, S. Mathis ${ }^{1,2}$ and A. Strugarek ${ }^{1}$
}

\begin{abstract}
This paper provides a brief overview of the formation of stellar fossil magnetic fields and what potential instabilities may occur given certain configurations of the magnetic field. One such instability is the purely magnetic Tayler instability, which can occur for poloidal, toroidal, and mixed poloidal-toroidal axisymmetric magnetic field configurations. However, most of the magnetic field configurations observed at the surface of massive stars are non-axisymmetric. Thus, extending earlier studies in spherical geometry, we introduce a formulation for the global change in the potential energy contained in a convectively-stable region for both axisymmetric and non-axisymmetric magnetic fields.
\end{abstract}

\section{Motivation}

In keeping with Jean Paul Zahn's long-standing interest in stellar transport mechanisms, and in the interest of extending some of his earlier work on the Tayler instability (Zahn et al. 2007), we wish to investigate a generalization both of that work as well as that of Tayler (1973) to nonaxisymmetric magnetic fields. Such an instability may be active in stars and may lead to the dissipation of certain magnetic field configurations. Thus, it is useful to ascertain which magnetic field configurations are stable against the Tayler instability as a potential means of constraining the possible morphologies of fossil magnetic fields. The fossil field hypothesis states that remnants of the field built during a star's birth persist in the radiative, convectively-stable zones of main-sequence stars (Alecian et al. 2013). This hypothesis is invoked to explain the large-scale magnetic field configurations observed at the surface of stars with a radiative exterior (Neiner et al.

\footnotetext{
${ }^{1}$ Laboratoire AIM Paris-Saclay, CEA/DRF - CNRS - Université Paris Diderot, IRFU/SAp Centre de Saclay, 91191 Gif-sur-Yvette Cedex, France; e-mail: kyle.augustson@cea.fr

2 LESIA, Observatoire de Paris, PSL Research University, CNRS, Sorbonne Universités, UPMC Univ. Paris 06, Univ. Paris Diderot, Sorbonne Paris Cité, 5 place Jules Janssen, 92195 Meudon, France
} 
(a)

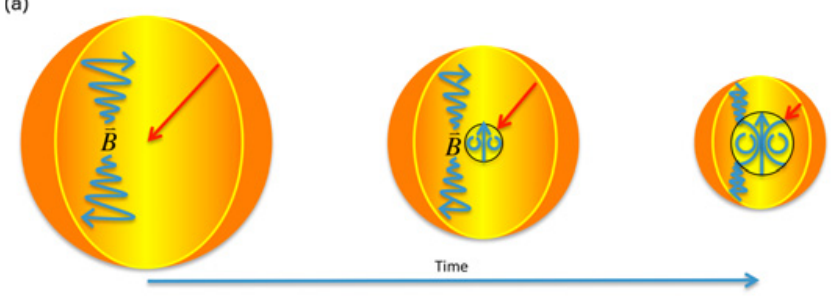

(b)

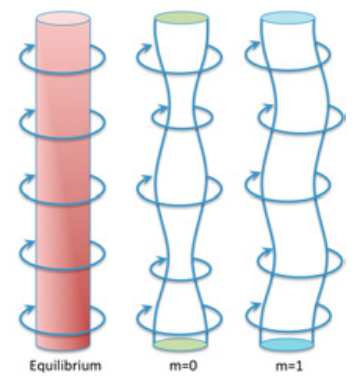

Fig. 1. (a) A sketch of the transition from an initially fully convective, pre-main-sequence star to a main-sequence star with a stable radiative interior. A convective dynamo establishes the fossil field. Once convection has halted, it relaxes into a stable configuration during the evolution of the stable region. The stellar magnetic field is a superposition of dynamo-generated and fossil fields. The red arrow denotes the contraction of the convective envelope, the convectively-stable core is the yellow region encircled by a black line. (b) Tayler instabilities in a cylindrical current channel. From left to right: shows an equilibrium configuration for an azimuthal magnetic field, a varicose $(\mathrm{m}=0)$ instability, and a kink type $(\mathrm{m}=1)$ instability. Field lines are marked with arrows.

2015; Braithwaite \& Spruit 2015). The magnetic field observed at the surface of massive stars is typically a non-axisymmetric oblique dipole or a similarly simple geometry (Moss et al. 1990; Walder et al. 2012; Wade et al. 2016). Given the hydrodynamic similarity of the stably-stratified regions of massive and low-mass stars, such non-axisymmetric magnetic fields may also exist within these regions for low-mass stars (Strugarek et al. 2011). Fossil magnetic fields have been proposed as an important source of angular momentum transport and mixing across the Hertzsprung-Russell diagram (e.g., Gough \& McIntyre 1998; Heger et al. 2005; Mathis \& Zahn 2005). Thus, constraining the stability of magnetic fields within the convectively-stable, radiative regions is important when characterizing their influence on the transport of angular momentum over evolutionary timescales. It is also useful to better understand the observed magnetic field geometry for intermediate and high mass stars and its consequences for the local stellar environment (e.g., Petit et al. 2012).

As an example of how fossil fields can form, Figure 1a depicts the process of freezing out the magnetic field as an initially fully convective star evolves along the pre-main-sequence (e.g., Emeriau-Viard \& Brun 2017), when the star undergoes gravitational contraction. Once convection has halted in the stably-stratified layers, the field will undergo a slow Ohmic decay if the field has a stable configuration or a fast Alfvénic decay if it is unstable. To determine which decay path the magnetic field will take, one can assess the stability of the system to small displacements of fluid elements. If the growth rate of those small perturbations is real and positive, the magnetic field undergoes the Tayler instability. 


\section{The Tayler instability}

The analyses of Tayler (1973) and Markey \& Tayler (1973) address the stability conditions for toroidal and poloidal configurations of an axisymmetric magnetic field within a quiescent, stably-stratified medium. Three examples of the local instabilities arising in systems with an axisymmetric magnetic field are sketched in Figure 1b. A stable equilibrium arises when the magnetic field has no associated current (e.g., if it is potential), or if the Tayler stability criterion are met. However, when the current density or Lorentz force are sufficiently large, two other instabilities can be excited: the axisymmetric $m=0$ varicose instability or the $m=1$ kink instability, which is the most rapidly growing mode of the two. An analysis of the instabilities indicates that only certain mixed (poloidal and toroidal) configurations of axisymmetric magnetic fields are stable within the radiative regions of stars (Tayler 1980; Braithwaite 2009). If one accounts for rotational effects, the characteristics of these axisymmetric systems are modified in that the Coriolis force enhances their stability (Pitts \& Tayler 1985). The nature of the equilibrium states of mixed-morphology magnetic fields has been considered extensively in both non-rotating and rotating systems (e.g., Prendergast 1956; Braithwaite \& Nordlund 2006; Braithwaite 2008; Duez \& Mathis 2010; Duez et al. 2010; Duez 2011; Braithwaite \& Cantiello 2013; Emeriau \& Mathis 2015).

The $\sigma$-stability analysis elaborated in Tayler (1973) can be generalized to global-scale geometries as shown in Goossens (1980). However, axisymmetric magnetic fields are only one component of the full magnetic field. Indeed, the analysis can be extended to configurations with both non-axisymmetric magnetic fields and differential rotation as established in an upcoming paper (Augustson et al. 2017). The resulting stability criteria aid in isolating the possible magnetic field configurations within the stable regions of stars, thereby restricting the routes of angular momentum transport and limiting the types of interactions with the dynamo-generated magnetic fields established in convective regions.

\section{Fundamental equations}

The linearized equation of motion under the Cowling approximation (Cowling 1941) for a fluid element in a general, but non-rotating, coordinate system is

$$
\rho \frac{\partial^{2} \boldsymbol{\xi}}{\partial t^{2}}=\frac{1}{4 \pi}[(\boldsymbol{\nabla} \times \delta \boldsymbol{B}) \times \boldsymbol{B}+(\boldsymbol{\nabla} \times \boldsymbol{B}) \times \delta \boldsymbol{B}]-\delta \rho \boldsymbol{\nabla} \Phi-\boldsymbol{\nabla} \delta P,
$$

where $\boldsymbol{\xi}$ is the displacement, $\boldsymbol{B}$ the magnetic field, $\rho$ the density, $P$ the pressure, $\Phi$ the gravitational potential. The Eulerian perturbations $\delta$ of those quantities follow directly from the continuity, pressure, and induction equations as

$$
\delta \rho=-\nabla \cdot(\rho \boldsymbol{\xi}), \quad \delta P=-\boldsymbol{\xi} \cdot \boldsymbol{\nabla} P-\gamma P \boldsymbol{\nabla} \cdot \boldsymbol{\xi}, \quad \delta \boldsymbol{B}=\boldsymbol{\nabla} \times(\boldsymbol{\xi} \times \boldsymbol{B}) .
$$


Therefore, one has that

$$
\begin{aligned}
\rho \frac{\partial^{2} \boldsymbol{\xi}}{\partial t^{2}} & =\mathcal{F}[\boldsymbol{\xi} ; \rho, P, \Phi, \boldsymbol{B}]=\boldsymbol{\nabla} \cdot(\rho \boldsymbol{\xi}) \boldsymbol{\nabla} \Phi+\boldsymbol{\nabla}[\boldsymbol{\xi} \cdot \boldsymbol{\nabla} P+\gamma P \boldsymbol{\nabla} \cdot \boldsymbol{\xi}] \\
& +[(\boldsymbol{\nabla} \times \boldsymbol{\nabla} \times(\boldsymbol{\xi} \times \boldsymbol{B})) \times \boldsymbol{B}+(\boldsymbol{\nabla} \times \boldsymbol{B}) \times(\boldsymbol{\nabla} \times(\boldsymbol{\xi} \times \boldsymbol{B}))]
\end{aligned}
$$

where $\gamma$ is the ratio of specific heats.

As in Bernstein et al. (1958), one can consider simple solutions to the above equation that take the form $\boldsymbol{\xi}=R e[\boldsymbol{\psi}(\mathbf{x}) \exp (i \omega t)]$, for which the equation of motion yields $-\omega^{2} \rho \boldsymbol{\psi}=\mathcal{F}[\boldsymbol{\psi}]$. It can be shown that the vector function $\mathcal{F}$ is selfadjoint. With a properly defined inner product for the solutions $\boldsymbol{\xi}$, one can see that the dispersion relationship for a general displacement in an arbitrary coordinate system is given by

$\omega^{2}=-\frac{\langle\boldsymbol{\psi}, \mathcal{F}[\boldsymbol{\psi}]\rangle}{\langle\rho \boldsymbol{\psi}, \boldsymbol{\psi}\rangle}=-\int \boldsymbol{\psi}^{*} \cdot \mathcal{F} d V\left[\int \rho \boldsymbol{\psi}^{*} \cdot \boldsymbol{\psi} d V\right]^{-1}=2 \Delta W\left[\int \rho \boldsymbol{\psi}^{*} \cdot \boldsymbol{\psi} d V\right]^{-1}$

where the integral is taken over the region of interest, which for stars are their convectively-stable zones.

The displacement is unstable if the change in the potential energy of the system $(\Delta W)$ is negative. In general, one finds that this energy can be split into three parts as $\Delta W=\Delta W_{L}+\Delta W_{B}+\Delta W_{P}$, with $\Delta W_{L}$ being the work due to Lorentz forces, $\Delta W_{B}$ being the work due to buoyancy, and with $\Delta W_{P}$ being the pressure work. To find general classes of magnetic fields that are stable in a given radiative region, one needs to be able to find an expression for the work that can be minimized. This is possible within the context of separable coordinate systems. For this work, the spherical coordinate system is used. Therefore, for compactness and expedience, $\boldsymbol{\psi}, \rho, P, \Phi$, and $\boldsymbol{B}$ are projected onto the spherical spin vector harmonics (SVH). The SVH are a complete orthonormal set of vector functions that are formed from specific combinations of the spherical harmonics and their derivatives (Varshalovich 1988). When the work integrands are expanded on the SVH basis, one can show that

$$
\Delta W_{L}=\int_{r_{b}}^{r_{t}} d r r^{2} \sum_{i=1}^{4} \sum_{\substack{\ell, m, \nu, \mu, \lambda \\ \ell_{i}, m_{i}, \nu_{i}}} \lambda, \mu L_{\substack{\ell_{1}, \ell_{2} \\ \nu_{1}, \nu_{2}}}^{m_{1}, m_{2}}\left(\psi_{\ell_{3}, \nu_{3}}^{m_{3}} B_{\ell_{4}, \nu_{4}}^{m_{4}}\right) \psi_{\ell, \nu}^{* m} \mathcal{J}_{\substack{\ell_{3}, m_{3}, \nu_{3} \\ \ell_{4}, m_{4}, \nu_{4}}}^{\ell_{\ell_{2}, m_{2}, \lambda}} \mathcal{J}_{\substack{\ell_{2}, m_{1}, m_{2}, \nu_{2} \\ \ell, m_{1}, \nu}}^{\ell,}
$$

where each $\ell$ ranges from zero to infinity, each $m$ ranges between $-\ell$ and $\ell$, and where $\nu, \mu$, and $\lambda$ range between -1 and 1 . The integral is taken between radii $r_{b}$ and $r_{t}$, which demark the bottom and top boundaries of the radiatively stable region. The $\mathcal{J}$ coefficients arise from the projection of the cross products of the SVH basis vectors in Equation 3.1 back onto the basis. The $L$ symbol is a function of radius that arises from the Lorentz force, and thus it is a second-order 
differential operator involving the radial functions of the displacement and the magnetic field as

$$
\begin{aligned}
\lambda, \mu L_{\substack{\ell_{1}, \ell_{2} \\
\nu_{1}, \nu_{2}}}^{m_{1}, m_{2}}= & -\frac{B_{\ell_{1}, \nu_{1}}^{m_{1}}}{4 \pi}\left[E_{\nu_{2}, \lambda}^{\ell_{2}, m_{2}} \frac{\partial^{2}}{\partial r^{2}}+\frac{F_{\nu_{2}, \lambda}^{\ell_{2}, m_{2}}}{r} \frac{\partial}{\partial r}+\frac{G_{\nu_{2}, \lambda}^{\ell_{2}, m_{2}}}{r^{2}}\right] \psi_{\ell_{3}, \nu_{3}}^{m_{3}} B_{\ell_{4}, \nu_{4}}^{m_{4}} \mathcal{I}_{\lambda, \mu} \\
& +\frac{1}{4 \pi}\left[D_{\nu_{1}, \lambda}^{\ell_{1}, m_{1}} \frac{\partial B_{\ell_{1}, \lambda}^{m_{1}}}{\partial r}+C_{\nu_{1}, \lambda}^{\ell_{1}, m_{1}} \frac{B_{\ell_{1}, \lambda}^{m_{1}}}{r}\right] \\
& \times\left[D_{\nu_{2}, \mu}^{\ell_{2}, m_{2}} \frac{\partial}{\partial r}+\frac{C_{\nu_{2}, \mu}^{\ell_{2}, m_{2}}}{r}\right] \psi_{\ell_{3}, \nu_{3}}^{m_{3}} B_{\ell_{4}, \nu_{4}}^{m_{4}},
\end{aligned}
$$

where the coefficient matrices $C, D, E, F$, and $G$ describe the projection of the curl and double curl operators onto the spin vector harmonic basis, and $\mathcal{I}$ is the unit tensor. Assuming that the star is spherically symmetric, namely that the gradient of the gravitational potential is only in the radial direction, then one has that $g=-\partial_{r} \Phi$. So, tackling the buoyancy work integral, it can be seen that

$$
\begin{aligned}
\Delta W_{B}= & \int_{r_{b}}^{r_{t}} d r r^{2} g(r) \sum_{\substack{\ell, m, \ell_{1} \\
\ell_{2}, m_{2}, \nu_{2}}} \frac{(-1)^{m_{2}}}{2 \ell+1}\left(\sqrt{\ell+1} \psi_{\ell, 1}^{* m}-\sqrt{\ell} \psi_{\ell,-1}^{* m}\right) \mathcal{M}_{\ell, \nu} \\
& \times\left[\mathcal{K}_{\ell_{1} ;-m, m_{2} \ell, \nu} \rho_{\ell_{1}}^{m_{2}-\nu_{2}} \psi_{\ell_{2}, \nu_{2}}^{m_{2}}\right],
\end{aligned}
$$

Similarly, the pressure work integral can be identified as

$$
\begin{aligned}
& \Delta W_{P}=\int_{r_{b}}^{r_{t}} d r r^{2} \sum_{\substack{\ell, m, \ell_{1} \\
\ell_{2}, m_{2}}} \frac{1}{\sqrt{(2 \ell+1)\left(2 \ell_{2}+1\right)}} \mathcal{M}_{\ell, \nu}\left[\psi_{\ell, \nu}^{* m+m_{2}}\right] \\
& \left.\left\{(-1)^{m+m_{2}} \gamma \mathcal{H}_{\ell, \ell_{1}, \ell_{2}}^{m, m_{2}} P_{\ell_{1}}^{m} \mathcal{M}_{\ell_{2}, \nu}\left[\psi_{\ell_{2}, \nu}^{m_{2}}\right]+\sum_{\nu_{1}} \psi_{\ell_{1}, \nu_{1}}^{m} \mathcal{N}_{\ell_{2}, \nu} \underset{\mathcal{K}_{\ell ; \ell_{2}, \nu}^{m, m_{2}}}{\ell_{\ell_{1}, \nu_{1}}} P_{\ell^{2}}^{m_{2}}\right]\right\}
\end{aligned}
$$

Here, $\mathcal{H}$ and $\mathcal{K}$ are coefficients related to the 3 - $\mathrm{j}$ and $6-\mathrm{j}$ symbols that arise from integrals over products of SVH that are then either projected onto the scalar spherical harmonics, which, along with $\mathcal{J}$, are closely related to those defined in Varshalovich D.A. et al. (1988) and Strugarek et al. (2013). Note also that

$$
\begin{gathered}
\mathcal{M}_{\ell, \nu}=\sqrt{\ell+1}\left(\frac{\partial}{\partial r}+\frac{\ell+2}{r}\right) \delta_{\nu, 1}-\sqrt{\ell}\left(\frac{\partial}{\partial r}-\frac{\ell-1}{r}\right) \delta_{\nu,-1}, \\
\mathcal{N}_{\ell, \nu}=\sqrt{\ell+1}\left(\frac{\partial}{\partial r}-\frac{\ell}{r}\right) \delta_{\nu, 1}-\sqrt{\ell}\left(\frac{\partial}{\partial r}+\frac{\ell+1}{r}\right) \delta_{\nu,-1} .
\end{gathered}
$$

\section{Conclusions}

Utilizing the projected form of $\Delta W$, stability criterion can be found for which the system with a chosen general magnetic field is linearly stable or unstable to 
an arbitrary displacement by integrating the terms with radial derivatives of $\boldsymbol{\psi}$ by parts and then explicitly minimizing the radial integrals with respect to $\psi$. This will be demonstrated more completely in an upcoming paper (Augustson et al. 2017). Applying such criterion to stellar radiative zones will permit the determination of the stability of certain classes of magnetic fields that have a broad range of non-axisymmetric components. Possibly, one may ascertain why the most common magnetic field configuration observed has the magnetic symmetry axis oblique to the stellar rotation axis.

K.C. Augustson and Stéphane Mathis acknowledge support from the ERC SPIRE 647383 grant. A. Strugarek acknowledges support from the Canadian Institute of Theoretical Astrophysics (National Fellow) and from the Canadian Natural Sciences and Engineering Research Council.

\section{References}

Alecian, E., Wade, G.A., \& Catala, C., et al., 2013, MNRAS, 429, 1027

Augustson, C., Mathis, S., \& Strugarek, A., 2017, A\&A, in prep.

Bernstein, I.B., Frieman, E.A., Kruskal, M.D., \& Kulsrud, R.M., 1958, Pro. R. Soc. London Ser. A., 244, 17

Braithwaite, J., 2008, MNRAS, 386, 1947

Braithwaite, J., 2009, MNRAS, 397, 763

Braithwaite, J., \& Cantiello, M., 2013, MNRAS, 428, 2789

Braithwaite, J., \& Nordlund, A., 2006, A\&A, 450, 1077

Braithwaite, J., \& Spruit, H.C., 2015, ArXiv e-prints

Cowling, T.G., 1941, MNRAS, 101, 367

Duez, V., 2011, Astronomische Nachrichten, 332, 983

Duez, V., Braithwaite, J., \& Mathis, S., 2010, ApJ, 724, L34

Duez, V., \& Mathis, S., 2010, A\&A, 517, A58

Emeriau, C., \& Mathis, S., 2015, in IAU Symposium, Vol. 307, New Windows on Massive Stars, ed. G. Meynet, C. Georgy, J. Groh, \& P. Stee, p. 373

Emeriau-Viard, C., \& Brun, A.S., 2017, ApJ, 846, 8

Goossens, M., 1980, Geophys. Astrophys. Fluid Dyn., 15, 123

Gough, D.O., \& McIntyre, M.E., 1998, Nature, 394, 755

Heger, A., Woosley, S.E., \& Spruit, H.C., 2005, ApJ, 626, 350

Markey, P., \& Tayler, R.J., 1973, MNRAS, 163, 77

Mathis, S., \& Zahn, J.-P., 2005, A\&A, 440, 653

Moss, D.L., Mestel, L., \& Tayler, R.J., 1990, MNRAS, 245, 550

Neiner, C., Mathis, S., Alecian, E., et al., 2015, in IAU Symposium, Vol. 305, Polarimetry, ed. K. N. Nagendra, S. Bagnulo, R. Centeno, \& M. Jesús Martínez González, p. 61

Petit, V., Owocki, S.P., Oksala, M.E., \& MiMeS Collaboration, 2012, in Astronomical Society of the Pacific Conference Series, Vol. 465, Proceedings of a Scientific Meeting in Honor of Anthony F.J. Moffat, ed. L. Drissen, C. Robert, N. St-Louis, \& A.F.J. Moffat, p. 48 
Pitts, E., \& Tayler, R.J., 1985, MNRAS, 216, 139

Prendergast, K.H., 1956, ApJ, 123, 498

Strugarek, A., Brun, A.S., \& Zahn, J.-P., 2011, Astronomische Nachrichten, 332, 891

Strugarek, A., Brun, A.S., Mathis, S., \& Sarazin, Y., 2013, ApJ, 764, 189

Tayler, R.J., 1973, MNRAS, 161, 365

Tayler, R.J., 1980, MNRAS, 191, 151

Varshalovich, D.A., Moskalev, A.N., \& Khersonskii, V.K., 1988, Quantum Theory of Angular Momentum, ed. V.K. Khersonskii (World Scientific)

Wade, G.A., Neiner, C., Alecian, E., et al., 2016, MNRAS, 456, 2

Walder, R., Folini, D., \& Meynet, G., 2012, SSR, 166, 145

Zahn, J.-P., Brun, A.S., \& Mathis, S., 2007, A\&A, 474, 145 